\title{
Elderly patient refractory to multiple pain medications successfully treated with integrative East-West medicine
}

\author{
Bill Tu \\ Michael Johnston \\ Ka-Kit Hui \\ UCLA Center for East-West \\ Medicine, Department of Internal \\ Medicine, UCLA David Geffen School \\ of Medicine, Los Angeles, CA, USA
}

Background: Polypharmacy is a common and serious problem in the elderly today. Few solutions have been effective in reducing its incidence.

Case summary: An 87-year-old female with a history of osteoarthritis and spinal stenosis presented with a five month history of severe right hip pain. She had been seen by multiple specialists and hospitalized many times. During these encounters, she was prescribed a long list of pain medications. However, these medications did not improve her pain and added to her risk of adverse drug events. After exhausting traditional Western medical therapies, she received a referral to the UCLA Center for East-West Medicine. There, clinicians treated her with a nonpharmacological integrative East-West medicine approach that included acupuncture, dry needling of trigger points, and education on self-acupressure. Her pain began improving and she was able to cut back on analgesic use under physician supervision. Ultimately, she improved to the point where she was able to discontinue all of her pain medications. Symptomatic relief was evidenced by improvement in health-related quality of life (HRQOL).

Conclusions: This case study suggests that integrative East-West medicine may have the potential to reduce the incidence of polypharmacy in elderly patients presenting with pain conditions and improve their quality of life.

Keywords: polypharmacy, pain, osteoarthritis, acupuncture, complementary and alternative medicine, integrative medicine, adverse drug reaction, elderly

\section{Introduction}

Polypharmacy is a growing problem in the elderly population today. It is commonly defined either by number (more than 5 or more medications; Satish et al 1996) or by indication (more than one medication than is clinically indicated; Carlson 1996). Office visits involving polypharmacy for elderly patients have nearly quadrupled from 10.1 million in 1990 to 37.5 million in 2000 (Aparasu et al 2005). In fact, the average elderly American is on approximately 9 medications (prescription and nonprescription) (Stewart and Cooper 1994). This presents a grave problem because polypharmacy is a risk factor for adverse drug reactions (ADRs; Nguyen et al 2006), now estimated to result in 106,000 fatalities per year (Lazaridou et al 1998). The frequency of adverse events increases in a linear fashion that is proportional to the number of medications used (Kohn et al 1999). This report examines the case of an elderly polypharmacy patient who was able to discontinue her pain medications through the use of integrative East-West medicine. This led not only to an elimination of the risk of medication-induced side effects and adverse events but also resulted in significant improvement in her health-related quality of life (HRQOL).

\section{Case report}

An 87-year-old female with a history of osteoarthritis and spinal stenosis presented to her primary care physician with severe right hip pain. The pain radiated down her 
right leg and had been progressively worsening over the previous month. She was begun on a course of nonsteroidal anti-inflammatory drugs (NSAIDs). However, despite the use of etodoloac and over-the-counter analgesics, she did not improve and became unable to walk. Because of this, the patient went to her local emergency room for care and after evaluation was hospitalized for severe intractable pain. A magnetic resonance image (MRI) taken during hospitalization showed pronounced degenerative disc disease and lumbar spondylosis from L2 to S1 with probable nerve impingement at L5 and S1. In the hospital, she was evaluated by both neurosurgery and pain medicine services. She was then given lumbar facet joint injections from L3 to $\mathrm{S} 1$ with triamcinolone and a sacroiliac injection. The patient was discharged to a nursing home on oxycodone/ acetaminophen, propoxyphene napsylate/acetaminophen, rofecoxib (25 mg QD), and cyclobenzaprine (10 mg TID). Shortly after discharge, despite all these interventions she was again admitted to the hospital for pain. During this second hospitalization, treatment included a right trochanteric bursa injection with triamcinolone along with an escalation of pain medications.

The patient's pain persisted after discharge, however, and so four months later she was referred to the University at California Los Angeles Center for East-West Medicine (UCLA CEWM). She was wheelchair-bound due to pain. The patient was seen at the CEWM for a series of 12 weekly visits during which acupuncture treatment was performed by a medical doctor. Sessions lasted approximately 25 minutes and usually involved four acupuncture points (LI 4, LIV 3, ST 36, and GB 31). Additionally, the doctor dry-needled trigger points in the gluteal and illiotibial band regions. Occasionally, minimal amounts $(\leq 0.3 \mathrm{cc})$ of $1 \%$ lidocaine were used. She and her caretaker were then taught self-acupressure to do at home. The patient was given exercise and stretching recommendations based on traditional Chinese medicine principles.

Following several visits, she began noting marked improvement in her symptoms. Also, her functional status improved. Instead of using a wheelchair, she began to ambulate with the aid of a walker. Her medication use began to decline. When she first arrived at the CEWM, the patient was taking oxycodone/acetominophen 7.5/500 and propoxyphene napsylate/acetaminophen 100/650 for her pain. In the month preceding her first visit at the Center, the patient had taken oxycodone/acetominophen 7.5/500 10 times and propoxyphene napsylate/acetaminophen 7 times, according to nursing home records. Table 1 shows a summary of her medication use.

As her symptoms improved, her pain medications were carefully titrated downwards. She was advised to decrease her usage of as needed pain medications as tolerated. At her five-month follow-up visit to the UCLA CEWM, the patient

Table I Patient use of pain medicines

\begin{tabular}{|c|c|c|c|}
\hline Usage sequence & Medicine & Dosage & Class \\
\hline \multirow[t]{3}{*}{ Prior to $10 / 05 / 03$} & Etodoloac & $300 \mathrm{mg}$ TID & NSAID \\
\hline & Over-the-counter analgesics & $650 \mathrm{mg}$ QID & Analgesic(Misc.) \\
\hline & (Ex:Acetominophen) & & \\
\hline \multirow[t]{4}{*}{ Hospitalization on $10 / 05 / 03$} & Lumbar facet joint injections, L4 to L5 & 20 mg Triam. & Steroid \\
\hline & (Triamcinolone/lidocaine) & 2.5 cc I\% Lido. & Anesthetic \\
\hline & Sacroiliac Injection & 20 mg Triam. & Steroid \\
\hline & (Triamcinolone/lidocaine) & 2.5 cc I\% Lido. & Anesthetic \\
\hline \multirow[t]{3}{*}{$\begin{array}{l}\text { Following discharge from hospital } \\
\text { on } 10 / 13 / 03\end{array}$} & Oxycodone/acetaminophen & $\begin{array}{l}\text { 7.5/500 TID prn } \\
\text { (took I0X/mo.) }\end{array}$ & Comb. Opiod \\
\hline & Propoxyphene napsylate/acetaminophen & $\begin{array}{l}\text { I00/650 QID prn } \\
\text { (took 7X/mo.) }\end{array}$ & Comb. Opiod \\
\hline & Rofecoxib & $25 \mathrm{mg}$ QD & COX 2 Inhibitor \\
\hline \multirow[t]{2}{*}{ Rehospitalized on 10/22/03 } & Right trochanteric bursa injection & 40 mg Triam. & Steroid \\
\hline & (Triamcinolone/lidocaine) & $5 c c$ I\% Lido. & Anesthetic \\
\hline \multicolumn{4}{|c|}{ Patient starts treatment at UCLA Center for Eawst-West Medicine } \\
\hline 12 treatments over six months & $\begin{array}{l}\text { Acupuncture, dry needling trigger points, } \\
\text { acupressure }\end{array}$ & None* & None \\
\hline
\end{tabular}

Notes: *Occasional 0.1 cc I\% lidocaine used (minimal amounts).

Abbreviations: Lido., lidocaine; NSAID, nonsteroidal anti-inflammatory drugs; Triam., triamcinolone; UCLA, University of California Los Angeles. 
reported a complete discontinuation of pain medication. This was confirmed by analysis of nursing home records. She had reduced her use of oxycodone/acetominophen 7.5/500 from 10 times to zero times and propoxyphene napsylate/ acetaminophen from 7 times to zero times. She required neither steroid injections by pain management nor additional hospitalization for pain.

In a subsequent visit to the pain medicine department, the specialist noted that, "The acupressure and acupuncture has helped her tremendously as well, and at this point she seems to be rather stable, and she would like to continue as she has been, and she is not desiring any injections at this point." It was noted that her pain had diminished and that her overall health was much improved.

The patient filled out a survey to assess the quality of life at the start of treatment at the UCLA CEWM and at the five-month follow-up. A standard instrument, the Medical Outcomes Study 36 Item Short-Form (SF36) Health Survey was used. There was a dramatic improvement in Pain scores from 25 to 46 points ( 50 being the score of a normal person). This represents an $84 \%$ improvement in pain over a period of five months. There were improvements in the other categories as well including Social Functioning (19 points), Emotional Functioning (13 points), and Physical Functioning (9 points). Overall, there was more than a two standard deviation improvement in Pain, nearly a two standard deviation improvement in Social Functioning, more than a one standard deviation improvement in Emotional Functioning, and nearly a one standard deviation improvement in Physical Functioning (Figure 1).

\section{Discussion}

This case provides evidence that integrative East-West medicine (the combination of traditional Chinese and Western medicines) can play a useful role as a first-line treatment for many types of chronic pain. Randomized controlled trials show that acupuncture may alleviate chronic low back pain, knee pain, and osteoarthritis (Berman et al 2004; White et al 2007; Ammendolia et al 2008). Those in favor of a role for acupuncture see this evidence as sufficient for action. Acupuncture is thought to work by stimulating the body's own pain relieving system resulting in the release of endogenous opiods and for this reason has fewer side effects than treatment with exogenous opiods (Pomeranz and Chiu 1976; Yamashita et al 1998). Those who see the current evidence as insufficient to warrant accepting a role for acupuncture in mainstream medicine make the point that the evidence from randomized controlled trials suggest that short-term treatment with acupuncture does not result in long-term benefits (Wang et al 2008). In the case presented here, treatment with acupuncture combined with titration of pain medication resulted in alleviation of pain documented

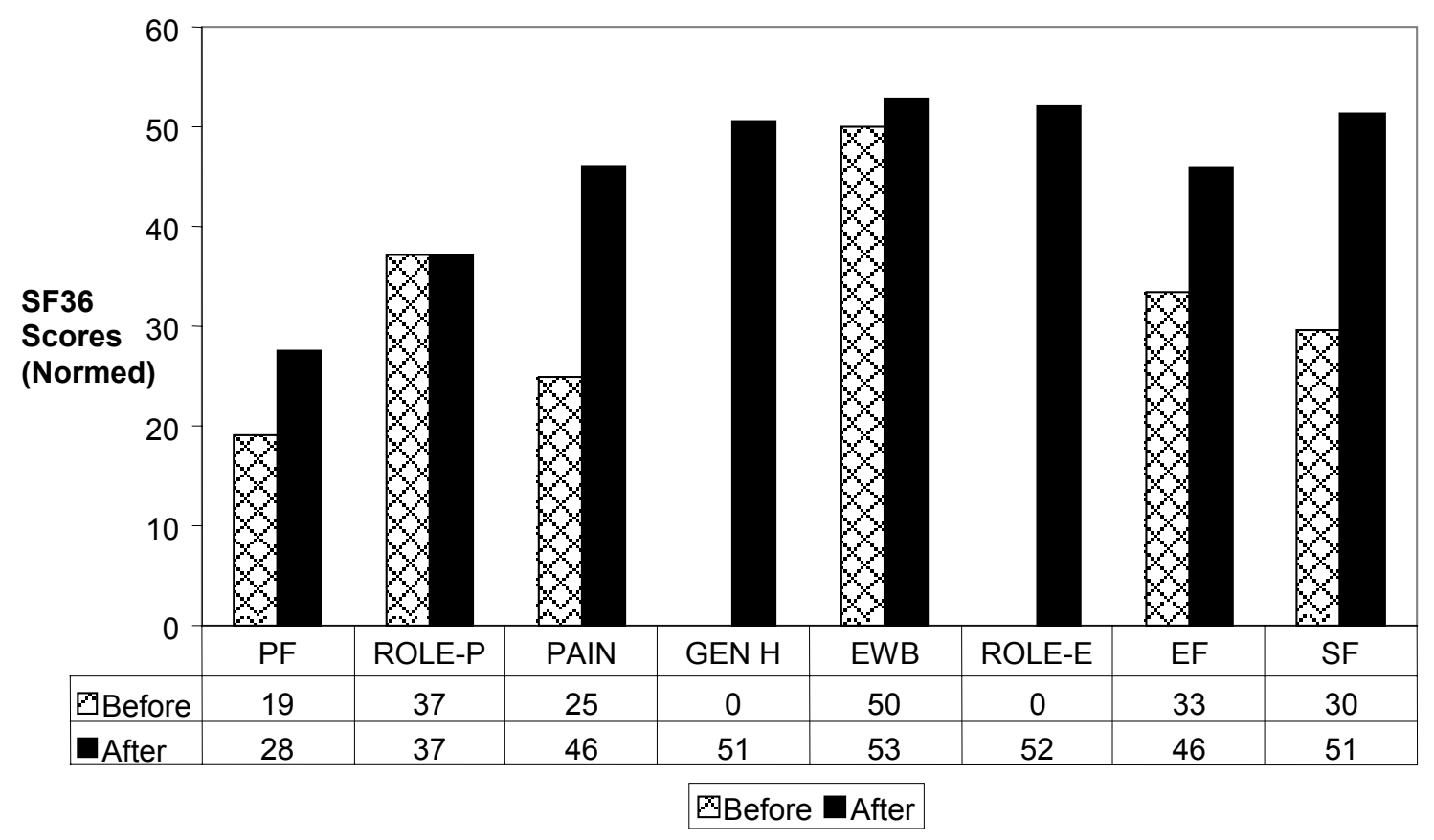

Figure I Quality of life. 
to have lasted at least five months beyond completion of the initial course of acupuncture therapy.

This case represents a common scenario encountered in the elderly in whom the treatment for a chronic condition such as osteoarthritic pain results in polypharmacy and unexpected deleterious effects to the patient's overall health. Side effects of pain medications include gastritis/gastrointestinal bleed (NSAIDs), increased cardiovascular event risk (COX-2 inhibitor), and physical dependence and mental status change (opiods). Acupuncture and similar modalities provide another plausible treatment option. Integrative medicine is the attempt to combine Western medicine with complementary and alternative medicine (CAM) in a system that emphasizes holistic, patient-centered care. CAM use is prevalent in the elderly population. In 2002 , as many as $27.7 \%$ of persons over 65 in the United States had used CAM (Arcury et al 2008). Among those adults with chronic diseases such as osteoarthritis 59.6\% reported the use of CAM (Saydah and Eberhardt 2006).

CAM includes several whole systems of medicine. One of the most widely practiced of these is traditional Chinese medicine. Integrative East-West medicine focuses the integration particularly of Western medicine with Chinese medicine. With regard to polypharmacy, this combination is ideal as the practitioner (trained in both Western and Eastern medicines) has the pharmacological background to safely titrate down medications, while simultaneously possessing the ability to provide the patient with nonpharmacological alternative treatments (ie, acupuncture).

Myofascial trigger points also often play a significant role in the pain related to osteoarthritis (Yentür et al 2004). Osteoarthritis may result in muscle spasm and trigger points in the areas surrounding the affected joint. Since there is significant overlap between myofascial trigger points and acupuncture points, this may be one of the means by which acupuncture benefits osteoarthritis patients (Melzack 1981). Dry-needling of trigger points, too, may be of help to pain patients, as was seen in this case (Hseih et al 2007). This technique allows for deactivation of a trigger point without exposure to the side effects of medication.

In conclusion, this case suggests that integrative EastWest medicine may be utilized effectively to reduce the incidence of polypharmacy in the elderly. Such an illustration is noteworthy because acupuncture is safe and medications for the treatment of myofascial pain carry the risk of side effects. In comparison, the risks associated with acupuncture are minimal (Yamashita et al 1998). Lowering the dose and/or number of pain medications in the elderly through integrative East-West medicine has much promise as a potential means for reducing the complications of polypharmacy. However, further research with larger number of subjects is warranted to confirm this finding.

\section{Acknowledgments}

The authors would like to thank Liz Logue of the UCLA David Geffen School of Medicine for her assistance in editing the paper. The authors report no conflicts of interest in this work.

\section{References}

Arcury TA, Suerken CK, Grzywacz JG, et al. 2006. Complementary and alternative medicine use among older adults: ethnic variation. Ethn Dis, 16:723-31.

Ammendolia C, Furlan AD, Imamura M, et al. 2008. Evidence-informed management of chronic low back pain with needle acupuncture. Spine $J$, $8: 160-72$

Aparasu RR, Mort JR, Brandt H. 2005. Polypharmacy trends in office visits by the elderly in the United States, 1990 and 2000. Res Social Adm Pharm, 1:446-59.

Berman BM, Lao L, Langenberg P, et al. 2004. Effectiveness of acupuncture as adjunctive therapy in osteoarthritis of the knee: a randomized, controlled trial. Ann Intern Med, 141:901-10.

Carlson JE. 1996. Perils of polypharmacy: 10 steps to prudent prescribing. Geriatrics 51:26-30, 35.

Hsieh YL, Kao MJ, Kuan TS, et al. 2007. Dry needling to a key myofascial trigger point may reduce the irritability of satellite MTrPs. Am J Phys Med Rehabil, 86:397-403.

Kohn L, Corrigan J, Donaldson M (eds). 1999. To Err is Human: Building a Safer Health System. Institute of Medicine. Washington, D.C.: National Academy Press.

Lazarou J, Pomeranz B, Corey P. 1998. Incidence of adverse drug reactions in hospitalized patients. JAMA, 279:1200-5.

Melzack R. 1981. Myofascial trigger points: relation to acupuncture and mechanisms of pain. Arch Phys Med Rehabil, 62:114-17.

Nguyen JK, Fouts MM, Kotabe SE, et al. 2006. Polypharmacy as a risk factor for adverse drug reactions in geriatric nursing home residents. Am J Geriatr Pharmacother, 4:36-41.

Pomeranz B, Chiu D. 1976. Naloxone blockade of acupuncture analgesia: endorphin implicated. Life Sci, 19:1757-62.

Satish S, Winograd CH, Chavez C, et al. 1996. Geriatric targeting criteria as predictors of survival and health care utilization. J Am Geriatr Soc 44:914-21.

Saydah SH, Eberhardt MS. 2006. Use of complementary and alternative medicine among adults with chronic diseases: United States 2002. J Altern Complement Med, 12:805-12.

Stewart RB, Cooper JW. 1994. Polypharmacy in the aged. Drugs Aging, 4:449-61.

Wang SM, Kain ZN, White PF. 2008. Acupuncture analgesia: II. Clinical considerations. Anesth Analg, 106:611-21.

White A, Foster NE, Cummings M, et al. 2007. Acupuncture treatment for chronic knee pain: a systematic review, Rheumatology (Oxford), 46:384-90.

Yamashita H, Tsukayama H, Tanno Y, et al. 1998. Adverse events related to acupuncture. JAMA, 280:1563-4.

Yentür EA, Okçu G, Yegül I. 2004. The role of trigger point therapy in knee osteoarthritis, The Pain Clinic, 15:385-90. 